\title{
The multi-needle Langmuir probe instrument for QB50 mission: Case studies of Ex-Alta 1 and Hoopoe satellites
}

\author{
H. Hoang - K. Røed - T. A. Bekkeng - J. I. Moen · \\ L. B. N. Clausen - E. Trondsen - B. Lybekk · H. \\ Strøm - D. M. Bang-Hauge - A. Pedersen - C. D. \\ A. Nokes · C. Cupido · I. R. Mann · M. Ariel · D. \\ Portnoy · E. Sagi
}

Received: date / Accepted: date

\begin{abstract}
The QB50 mission is a satellite constellation designed to carry out measurements at between $200-380 \mathrm{~km}$ altitude in the ionosphere. The multi-needle Langmuir probe (mNLP) instrument has been mounted on board eleven QB50 satellites in order to characterize ambient plasma. The distinct feature of this instrument is its capability of measuring the plasma density at high spatial resolution without the need to know the electron temperature or the spacecraft potential. While the instrument has been deployed on many sounding rockets, the QB50 satellites offer the opportunity to demonstrate the operation of the instrument in low-earth orbit (LEO). This paper provides a brief review of the m-NLP instrument specifically designed for the QB50 mission and the case studies of the instrument's performance on board the Ex-Alta 1 and Hoopoe satellites. The system has also been functionally verified in a plasma chamber at the European Space Research and Technology Center (ESTEC). Although the QB50 mission's scientific goals have not been reached yet and some uncertainties still remain, there are some optimistic in-orbit preliminary results which could be helpful for the system improvement in future campaigns. Particularly, the electron emitter as part of the m-NLP science unit has demonstrated its capability in the plasma chamber and in orbit to mitigate spacecraft charging effects.
\end{abstract}

Keywords QB50 · Langmuir probe · electron density · spacecraft charging

\section{Introduction}

A CubeSat is a small satellite standard initiated by California Polytechnic State University (Cal Poly) in collaboration with Stanford University's Space System Development Labo-

H. Hoang · K. Røed · T. A. Bekkeng · J. I. Moen · L. B. N. Clausen · E. Trondsen · B. Lybekk · H. Strøm · D. M. Bang-Hauge $\cdot$ A. Pedersen

Department of Physics, University of Oslo, 0316 Oslo, Norway

E-mail: huy.hoang@fys.uio.no

C. D. A. Nokes · C. Cupido · I. R. Mann

Department of Physics, University of Alberta, AB, Canada

M. Ariel · D. Portnoy · E. Sagi

Herzliya Science Center, Israel 
ratory in 1999. In the CubeSat specification, satellites are described in multiples of $1 \mathrm{U}$, where $1 \mathrm{U}$ has a volume of $10 \mathrm{~cm} \times 10 \mathrm{~cm} \times 10 \mathrm{~cm}$ cube with a maximum weight of $1.33 \mathrm{~kg}$. Typical CubeSats have sizes from $1 \mathrm{U}$ to $4 \mathrm{U}$. The project goal was to provide both mechanical and electrical specifications for a standard satellite platform. Additionally, a Poly-PicoSatellite Orbital Deployer (P-POD) was specifically developed to make it easy and secure for a launch provider to include CubeSat satellites aboard a rocket in addition to the rocket's main payload. This kind of standardization makes a CubeSat launch much cheaper than other regular launches of non-standardized satellites, paving the way to scientific investigations and technology demonstrations in low-earth orbit (LEO) in such a way that is cost-effective, timely and relatively easy to accomplish. The use of CubeSats has been increasingly exploited for commercial services and business, e.g. to support the Automatic Identification System (AIS) in the maritime sector (Helleren et al, 2012; Beattie et al, 2013), as well as for military applications (Venturini et al, 2009). The number of universities developing CubeSats has increased over the past decade. The complete set of CubeSat specifications is available online (Program, 2015).

QB50 is a project within the European Union's Seventh Framework Programme for Research (FP7) that aimed at launching a constellation of 50 CubeSats into the lower thermosphere at approximately $380 \mathrm{~km}$ altitude (Muylaert et al, 2009). One of the main purposes of the QB50 project is to achieve sustained and affordable access to space for small scale research space missions and planetary exploration. The QB50 satellites are expected to operate for three up to twelve months before burning up during re-entry. All QB50 satellites can carry their own instruments, but most of the satellites additionally accommodate a set of common science instruments to accomplish atmospheric research in the lower thermosphere. There are three different types of science instruments, each of which is part of the common instrument set. The Ion-Neutral Mass Spectrometer (INMS) is part of set 1 . The Flux- $\Phi$-Probe Experiment (FIPEX) is part of set 2 and the multi-needle Langmuir probe (m-NLP) science unit is part of set 3. A surface thermal monitor (STM) is installed in all satellites.

This paper will present the m-NLP science unit which has been built at the University of Oslo (UiO) (Bekkeng et al, 2010; Jacobsen et al, 2010). The main purpose of this paper is to provide a description of the instrument and to present first in-flight results from a system and technology demonstration point of view. The science unit includes four needle Langmuir probes and an electron emitter. The probe system had been deployed on many sounding rockets before the QB50 mission to resolve ionospheric plasma density variations down to the meter scale. In order to adapt to the limited resources in terms of size, power consumption and data-link budget in the QB50 satellites, instrument design adaptations have been made. Until now we have received the m-NLP data from three QB50 satellites including Experimental Albertan satellite number 1 (Ex-Alta 1), Hoopoe and Pegasus. While in this paper the data from the Ex-Alta 1 and Hoopoe satellites are discussed, the data from the Pegasus satellite will be reported in another paper. This paper is organized as follows. Section 2 describes the theoretical background and scientific objectives. A detailed description of the m-NLP science unit is introduced in the section 3, which also presents the system verification in a plasma chamber at the European Space Research and Technology Center (ESTEC). Section 4 presents some in-orbit preliminary results with two case studies of the Ex-Alta 1 and Hoopoe satellites, followed by some conclusions in section 5. 


\section{Background and scientific objectives}

\subsection{Background}

Since the initial work by Mott-Smith and Langmuir in the 1920s (Mott-Smith and Langmuir, 1926), Langmuir probes have been widely used as diagnostic instruments for both laboratory and space plasma (Boggess et al, 1959; Chen, 1965; Brace et al, 1973; Chapkunov et al, 1976; Brace, 1998). Traditionally, Langmuir probe operation is based on sweeping the bias voltage of a conductor exposed to a plasma and measuring the collected currents from the conductor. With the general understanding of the processes involved in the plasma, Langmuir probe theory is applied to infer the plasma parameters through the obtained currentvoltage (I-V) curve. However, sweeping takes time at least hundreds of ms and makes this approach unsuited for high spatial resolution measurements on space platforms.

Instead of sweeping the bias voltage, the m-NLP design (Jacobsen et al, 2010; Bekkeng et al, 2010) uses several cylindrical probes biased at different fixed voltages within the electron saturation region such that the currents to these probes can be sampled at a much higher rate, resulting in high-resolution plasma density observations. For a probe with its radius much smaller than the Debye length of a non-drifting, unmagnetized, and collisionless plasma, the electron saturation current to the probe can be described by the Orbital-MotionLimited (OML) theory as follows:

$$
I_{e}=C I_{e 0}\left(1+\frac{e V}{k_{B} T_{e}}\right)^{\beta}
$$

where $C=2 / \sqrt{\pi}$ for a cylindrical geometry and $C=1$ for planar and spherical geometries of the probes. $I_{e 0}=N_{e} A_{e} e \sqrt{\frac{k_{B} T_{e}}{2 \pi m_{e}}}$, is the electron thermal current to the probe at the plasma potential, $N_{\mathrm{e}}$ is the electron density, $k_{\mathrm{B}}$ is the Boltzmann constant, $T_{\mathrm{e}}$ is the electron temperature, $e$ is the electron charge, $A_{e}$ is the probe surface area, $m_{\mathrm{e}}$ is the electron mass and $V$ is the probe bias potential $V_{b}$ with respect to the plasma potential $V_{p}$ i.e. $V=V_{b}-V_{p}$. On space platforms, a probe bias is relative to a common electrical ground, which is connected to the spacecraft chassis so the signal ground is at the same level of the floating potential of the spacecraft chassis. Thus the parameter $V$ in Eq. 1 can be expressed by $V=V_{b}+V_{f}$, where the spacecraft floats relative to the plasma at a bias of $V_{f}$ and $V_{b}$ is the probe bias with respect to this spacecraft floating potential. The parameter $\beta$ is equal to $0,0.5$ and 1 for a planar, cylindrical and spherical probe, respectively. The electron density derived by the $\mathrm{m}-\mathrm{NLP}$ instrument deploying two cylindrical probes is given by:

$$
N_{e}=\frac{1}{K A_{e}} \sqrt{\frac{\Delta\left(I_{c}^{2}\right)}{\Delta V_{b}}}
$$

where $K$ is a constant given by $\frac{e^{3 / 2}}{\pi} \sqrt{\frac{2}{m_{e}}}, \Delta\left(I_{c}^{2}\right)$ is the difference in the square of collected currents and $\Delta V_{b}$ is the difference in the probe biases (Jacobsen et al, 2010). Under certain conditions, the m-NLP is also capable of monitoring the spacecraft potential and its variations as described in Bekkeng et al (2013).

Since the $\beta$ parameter is prone to variations, an alternative data analysis technique is using a method of non-linear least squares when fitting the probe measurement currents with the model shown in Eq. 1 i.e. with $\beta, N_{e}$ and $T_{e}$ being free parameters of the fitting 
process. The two approaches of estimating the electron density using Eq. 2 and the nonlinear least squares fit have advantages and disadvantages with respect to the current $\mathrm{m}$ NLP implementation, and both can be applied to derive the plasma parameters (Hoang et al, 2018).

\subsection{Scientific objectives}

The m-NLP probe system was originally developed for the Investigation of Cusp Irregularities (ICI) program and it was first flown on the ICI-2 sounding rocket in 2008 (Moen et al, 2012; Oksavik et al, 2012; Spicher et al, 2014, 2015b). Our basic research desire is to explore the chain of multiscale physical processes giving rise to ionospheric scintillations (Moen et al, 2013, and references therein). Radio communication and Global Navigation Satellite Systems (GNSS) both suffer from ionospheric scintillations, and the problems are most severe at low and high latitudes (e.g. Basu and Basu, 1981; Basu et al, 1988b,a, 1990, 1998). In GNSS ionospheric systems, scintillations can cause rapid variations of the measured phase of a signal, causing degraded positioning accuracy. These problems may take place during the entire solar cycle (Kintner et al, 2007). Prikryl et al (2010) reported serious GNSS signal degradation in the polar cusp even during solar minimum conditions. Unless ionospheric scintillation forecasts to reliably predict the signal quality become available, users in the affected regions cannot fully rely on communication and navigation services without taking risk. The m-NLP system on a fleet of CubeSats is expected to be the most cost effective approach to monitor the dynamics of the problem regions and hence be valuable to any attempts to regional or global forecast services.

The idea with QB50 was to deploy several spacecraft like pearls on a string, which would be ideal to revisit instability regions and to study growth and decay of instability regions, as was demonstrated during the commissioning of the Swarm mission (Spicher et al, 2015a; Goodwin et al, 2015). In addition to provide plasma density measurements, the main drivers for $\mathrm{UiO}$ to get involved with the QB50 mission are:

- Demonstrate the m-NLP system can provide high resolution electron density measurements on board CubeSats.

- Explore temporal evolution of F-region plasma irregularities in the polar caps and in the equatorial region.

\section{The m-NLP science unit description and system verification}

\subsection{Instrument design for QB50 satellites}

We have adapted the m-NLP design for being possibly deployed on the QB50 satellites by: (i) lowering the power consumption, (ii) introducing onboard data processing (due to telemetry constraints), and (iii) developing an electron emitter to control the spacecraft potential (Bekkeng et al, 2017).

\subsubsection{Probe and boom system design}

The m-NLP science unit includes four Langmuir probes, which are made from a coaxial cable with bootstrapped sections to mitigate edge effects. Since the spacecraft conductive 


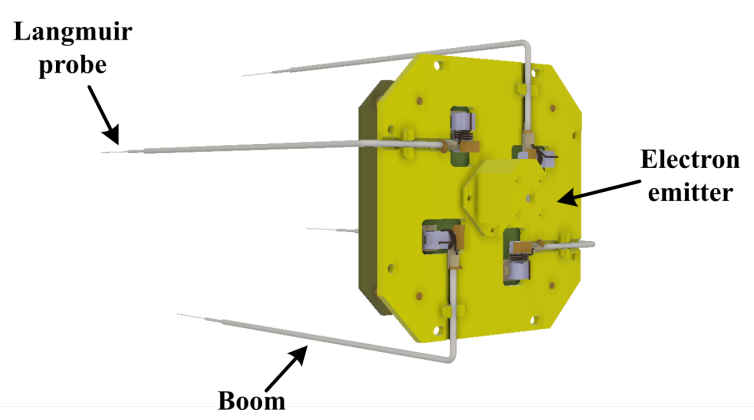

Fig. 1 CAD drawing of the m-NLP science unit, where the booms are in stowed position. The spring-loaded booms are designed to be stowed along the outer surface of the QB50 spacecraft, with a length of about 188 mm including the probe tip and its bootstrapped section.

surface area of CubeSats is much smaller than that of sounding rockets, the probe diameter was reduced from $0.51 \mathrm{~mm}$ for the sounding rocket version to $0.29 \mathrm{~mm}$, to alleviate the effect of spacecraft charging. Each of the m-NLP probes is $25 \mathrm{~mm}$ in length and has the bootstrapped section of $1.19 \mathrm{~mm}$ in diameter and $15 \mathrm{~mm}$ in length. The probe design specification ensures that the probe radius is much smaller the Debye length, which is ranging from a few $\mathrm{mm}$ to tens of $\mathrm{mm}$ in LEO, for the OML condition fulfillment.

One of the main design challenges of the m-NLP instrument aboard the QB50 satellites was getting the Langmuir probes in an area of undisturbed space plasma. When the spacecraft travels in space, it creates a 'plasma wake' in the opposite direction of travel similar to the disturbed region behind a moving vessel. Because this 'plasma wake' is poorly understood and hard to predict, the probes were placed on long booms, aiming at locating these probes as far out into the undisturbed plasma as possible. Thus the boom system, consisting of four separated booms, was designed to be deployable and mounted on a common Alodine $1200 \mathrm{~S}$ coated top plate of the m-NLP science unit. The spring-loaded booms are stowed along the outer surface of QB50 spacecraft, with a length of about $188 \mathrm{~mm}$ including the probe tip and its bootstrapped section. The boom length is limited to the size of the spacecraft itself. A computer-aided design (CAD) drawing of the m-NLP science unit is shown in Figure 1, where the booms are in stowed position. Each boom has a diameter of $2.2 \mathrm{~mm}$ and is made of aluminium. All the probes, bootstrapped supports and booms are coated by Aerodag $\mathrm{G}$ to provide a uniform material work function. Each boom has an individual hold down and release mechanism (HDRM) as shown in Figure 2. The spring-loaded booms are forced to stay stowed by using burn wires, which are pressed down onto two resistors with good thermal contact. The current needed to burn each of the wires is about $0.5 \mathrm{~A}$ at 5.0 $\mathrm{V}$ for 3 seconds. The preloaded spring mechanisms can be released on orbit via a ground command, allowing them to perform a $90^{\circ}$ turn, and consequently push the probes out to reach their deployed position.

\subsubsection{Electronics design}

All electronics are fitted on two circuit boards, front-end and digital PCBs, placed under the Alodine coated top plate. While the m-NLP electronics design for the QB50 satellites is not radiation hardened, a Microsemi ProASIC3L flash-based Field Programmable Gate Array (FPGA) has been used instead of an SRAM-based FPGA for an improvement of the 


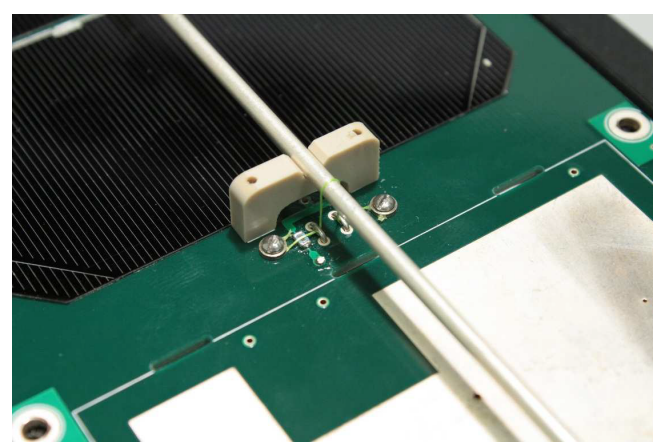

Fig. 2 Boom hold down and release mechanism (HDRM).

Table 1 Summary of the QB50 m-NLP instrument.

\begin{tabular}{ll}
\hline Mass & $\begin{array}{l}220 \mathrm{gr} \text { (including the boom system } \\
\text { and the electron emitter) }\end{array}$ \\
\hline Current measurement range & $1 \mathrm{nA}-2.2 \mu \mathrm{A}$ \\
\hline Current resolution & $1 \mathrm{nA}$ \\
\hline $\begin{array}{l}\text { Electron density range }\left(V_{b}=2 V \text { and }\right. \\
\left.T_{e}=1000 K\right)\end{array}$ & $2 \times 10^{9} \mathrm{~m}^{-3}-4.5 \times 10^{12} \mathrm{~m}^{-3}$ \\
\hline Output data resolution & $\begin{array}{l}16-\text { bit raw data (can down-sample } \\
\text { to } 14-, 12-, 10-\text { bit for more data) }\end{array}$ \\
\hline Sampling rate & \begin{tabular}{l} 
Up to $255 \mathrm{~Hz}$ \\
\hline Power consumption
\end{tabular} \\
$\begin{array}{l}\sim 1.1 \mathrm{~W} \text { (including the electron } \\
\text { emitter and real-time processing } \\
\text { power for electron density and } \\
\text { spacecraft potential computation) }\end{array}$ \\
\hline
\end{tabular}

radiation tolerance in the LEO environment. Details about the system electronics block are given in Bekkeng (2017). The power consumption has reduced from $3 \mathrm{~W}$ of the sounding rocket version to about $1 \mathrm{~W}$, which meets the power constraint for the QB50 satellites. The sampling rate can be set to upto $255 \mathrm{~Hz}$, however, the downlink budget of approximately 2 Mbits per day for the QB50 satellites severely limit the rate the amount of measurement time of the m-NLP instrument. The data length is set by default at 16-bit, but can be reduced to 14-, 12- and 10-bit to deal with the limited bandwidth. The fabricated m-NLP science unit is shown in Figure 3, where the booms are in their deployed position. It is noted that the instrument signal ground is electrically connected to the satellite platform. Table 1 presents a summary of the m-NLP developed for the QB50 mission.

The m-NLP instrument has two main operation modes, a fixed-bias mode and a sweptbias mode. While in the fixed-bias mode the probes are biased at fixed voltages for highresolution electron density measurement, the probes bias are swept from $-10 \mathrm{~V}$ to $10 \mathrm{~V}$ with 21 evenly spaced voltage steps in the sweep mode. The time between two adjacent voltage steps can be adjusted from $1 \mathrm{~ms}$ to $255 \mathrm{~ms}$. The fixed-bias mode is the mode during normal operation. The swept-bias mode can be used for probe performance verification during operations. 


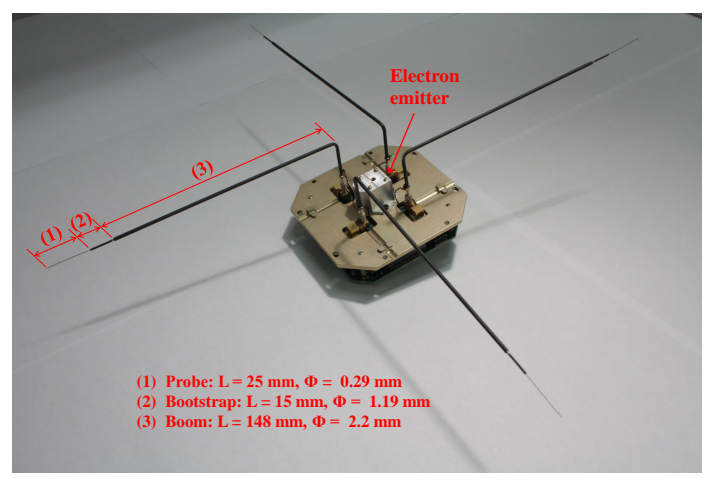

Fig. 3 Fabricated m-NLP science unit for QB50, where the booms are in their deployed position. The probe is $25 \mathrm{~mm}$ long with a $0.29 \mathrm{~mm}$ diameter. The bootstrapped section has a length of $15 \mathrm{~mm}$ and a diameter of $1.19 \mathrm{~mm}$. Figure adapted from Bekkeng (2017).

\subsubsection{Miniaturized thermionic electron emitter (MTEE)}

The four probes of the m-NLP instrument by default are positively biased with respect to the spacecraft payload potential, in order to draw electron currents from the ambient plasma that are then used to determine the electron density. Current continuity thus requires a return ion current through conductive surface of the spacecraft bus that are connected to the power ground. Since the ion current density is much smaller than the electron current density, either the spacecraft conductive surface is expected to be sufficiently large to neutralize coming electrons through the probes or the spacecraft platform would charge to highly negative potential values. As many of the QB50 satellite skin parts are not metallic, their conductive surfaces are very limited, for example, the total conductive surface area of Ex-Alta 1 is at most $120 \mathrm{~cm}^{2}$. In order to address this issue, an electron emitter was developed and encased in the housing mounted on the top plate of the m-NLP science unit as shown in Figure 3.

Electrons emitted from a heated filament are accelerated by a anode with its positive potential (accelerating voltage). The anode has a rather large aperture of $3 \mathrm{~mm}$, which prevents the majority of the electrons constituting the beam from being collected at the anode. As the electrons will get an outwards velocity when passing through the aperture of the anode, an additional electrode with an aperture of $3.2 \mathrm{~mm}$ is placed $1 \mathrm{~mm}$ in front of the anode. The purpose of this electrode is to limit the beam angle by collecting the most deflected electrons. The electrode was placed at the same potential as the anode to otherwise not affect the electron beam. More details of the electron emitter development can be found in Bekkeng et al (2017). Test results of the electron emitter have been obtained, showing that with a limited power consumption of $100 \mathrm{~mW}$, emitted beam currents could reach up to $54 \mu \mathrm{A}$ for the accelerating voltage of $38 \mathrm{~V}$ on the QB50 satellites (Hoang et al, 2017). Electrons emerge from the electron emitter with a kinetic energy of $38 \mathrm{eV}$, which corresponds to about 3600 $\mathrm{km} / \mathrm{s}$ with respect to the satellite. Thus the electron recollection probability is expected to be small. Assuming no negative spacecraft charging and ram ion current, an upper limit for the required emitter current can be estimated of the sum of the probe currents. For a 1000 $\mathrm{K}$ plasma with density of $10^{10} \mathrm{~m}^{-3}$, and biases of $1 \mathrm{~V}, 1.5 \mathrm{~V}, 2 \mathrm{~V}$, and $2.5 \mathrm{~V}$, the upper limit is estimated about $20 \mathrm{nA}$ using Eq. 1 . At a density of $10^{12} \mathrm{~m}^{-3}$, the maximum current needed is about $2 \mu \mathrm{A}$, presumably well within the capability of the emitter. Table 2 presents a summary of the electron emitter developed for the QB50 mission. 
Table 2 Summary of the electron emitter developed for the QB50 mission.

\begin{tabular}{ll}
\hline Type & Thermionic emission \\
\hline Accelerating voltage & $38 \mathrm{~V}$ \\
\hline Filament voltage range & $0.9-1.2 \mathrm{~V}$ \\
\hline Emitted current range & $20 \mathrm{nA}-54 \mu \mathrm{A}$ \\
\hline Power consumption & Up to $100 \mathrm{~mW} @ 1.2 \mathrm{~V}$ filament voltage \\
\hline
\end{tabular}

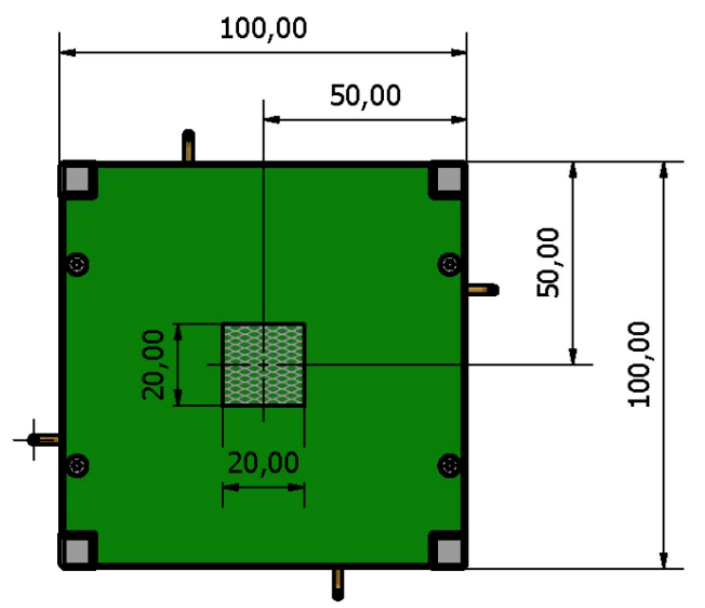

Fig. 4 STM TH0 placement on QB50 spacecraft wake-side panel ( $+Z$ face). The four protruding parts are the $\mathrm{m}-\mathrm{NLP}$ boom system viewed from the $+\mathrm{Z}$ face. All dimensions are given in $\mathrm{mm}$.

\subsubsection{Surface thermal monitor (STM)}

Besides the electron density and spacecraft charging measurement by the m-NLP science unit, there are six temperature transducers $\mathrm{AD} 590 * \mathrm{~F}$, namely $\mathrm{TH} 0$ to $\mathrm{TH} 5$, to monitor the temperature on the QB50 satellites. The output of each transducer is a twisted pair of wires without shielding. Five of those transducers, TH0 to TH4, are used to keep track of the temperature on different panels of the satellites, i.e. inner side of solar panels, and TH5 examines the temperature inside the m-NLP electronics board. Figure 4 shows placement of TH0 on the $+\mathrm{Z}$ face of the QB50 spacecraft. The QB50 spacecraft body coordinate system can be found in Denis (2015). The transducers TH1 to TH4 are located in the $-\mathrm{Y},-\mathrm{X},+\mathrm{Y}$ and $+X$ face, respectively. The $\mathrm{m}-\mathrm{NLP}$ science unit reads the temperature values from the transducers and periodically sends data to the onboard computer (OBC) every 3.5 minutes by default. The sampling rate of the STM experiment can be adjust via a ground command.

\subsubsection{Instrument interfaces to QB50 spacecraft}

The m-NLP science unit has been designed to mechanically interface to commercially available CubeSat structures either through an adapter or through appropriate relocation of mounting holes on the structures. The instrument was accommodated at the $-\mathrm{Z}$ face, which is ram velocity direction, of the QB50 spacecraft. Figure 5 illustrates a rendering model of ExAlta 1 (Mann and et. al., 2018), one of the QB50 satellites, carrying the m-NLP instrument 


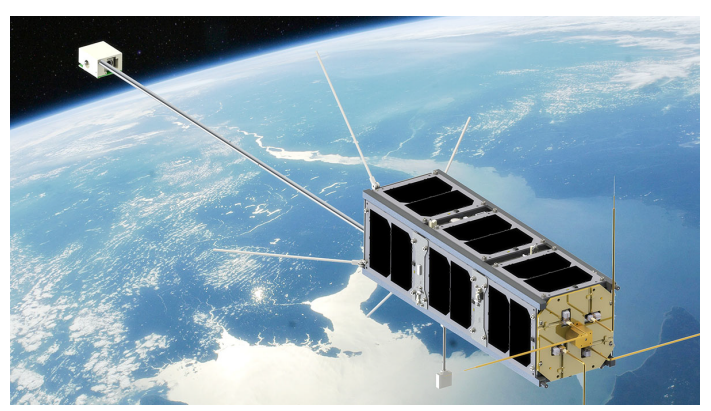

Fig. 5 QB50 Ex Alta-1 satellite.

in front. The m-NLP science unit goes $36 \mathrm{~mm}$, including the connector, downwards into the QB50 CubeSat structure, from the bottom side of the science unit top plate. In addition to the m-NLP science unit, the Ex-Alta 1 satellite carries a digital fluxgate magnetometer (FGM) (Miles et al, 2013, 2016), which is used for high frequency measurements of the Earth's magnetic field. The magnetometer is encased in a box mounted on a long boom behind the satellite.

The QB50 platform supplies regulated voltages 3.3 V and 5.0 V to the m-NLP science unit. The two power rails should achieve stabilized nominal voltage levels within maximum $150 \mathrm{~ms}$ after switch-on. A $3.3 \mathrm{~V}, 9600 \mathrm{bps}$ universal asynchronous receiver-transmitter (UART) serial interface is used to control the science unit from the OBC.

\subsubsection{Instrument script handling}

The OBC can execute scripts to operate the m-NLP instrument. The scripts provide a timetagged sequence of commands to be run. The m-NLP state transition is quite complicated, for instance, measurement data collection can not be executed until voltages and currents of the power rails are correctly verified and the probe bias is set. There is no broadcast mode on the QB50 m-NLP science unit, hence a data request command is sent whenever the measurement data collection is needed. As a consequence, the script size is relatively large, e.g. a script to turn on the instrument, ask for the housekeeping data including voltages and currents of the system power rails and then turn the instrument off is 42-byte long. A typical script with a common procedure to send a few data request commands would be more than 500-byte long.

It is possible to get either raw data (21 current measurements from each of the 4 probes) or the processed data ( 81 electron density and 8 spacecraft floating potential values) from the m-NLP science unit for each data request command.

\subsection{System functional verification in plasma chamber}

We have functionally verified the m-NLP science unit system in the plasma chamber at the European Space Research and Technology Center (ESTEC). The unit was mounted on a 2U CubeSat, where its solar cells were not installed. The solar panel locations were instead filled with conducting metallic patches. The total conductive surface area of the CubeSat is estimated to be about $450 \mathrm{~cm}^{2}$, including the Alodine coated top plate. The test setup is shown in Figure 6, where the m-NLP science unit is located on the ram-facing side of 


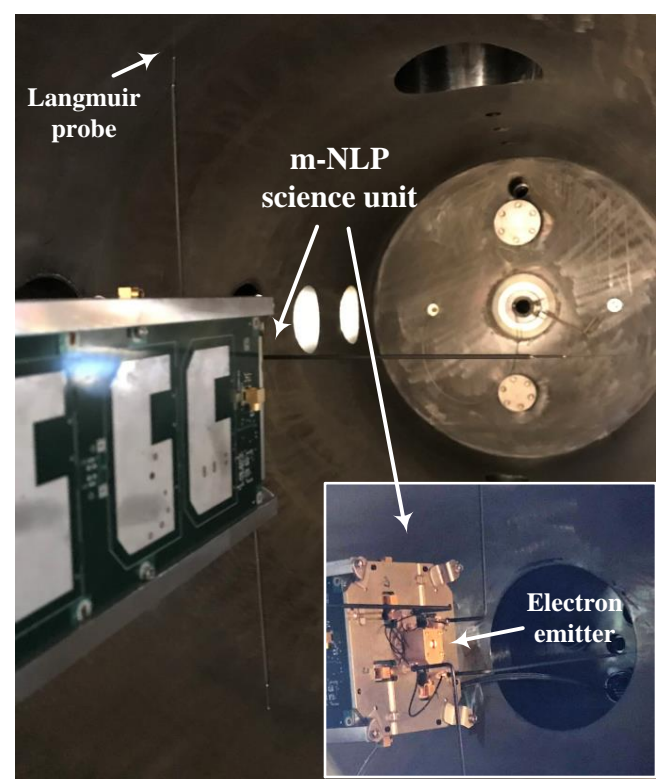

Fig. 6 Verification setup in the ESTEC plasma chamber. The total conductive surface area of the CubeSat is about $450 \mathrm{~cm}^{2}$, including the Alodine coated top plate. The bottom right conner of the figure shows the $\mathrm{m}-\mathrm{NLP}$ science unit with the electron emitter being in operation.

the CubeSat. The CubeSat was hung from the top of the chamber. This chamber is $1 \mathrm{~m}$ in diameter and $2 \mathrm{~m}$ in length, with a plasma source generating argon ions accelerated to approximately $7 \mathrm{~km} / \mathrm{s}$. Electrons in the chamber are generated by a heated filament, and flow down the centerline of the chamber together with the argon ions.

The effect of using the electron emitter is clearly shown in Figure 7. The m-NLP system operated in the swept-bias mode, where all of the m-NLP probes were swept simultaneously from $-10 \mathrm{~V}$ to $10 \mathrm{~V}$ with 21 steps and back to $-10 \mathrm{~V}$ with the same number of steps. The probes bias, which was swept with several step durations longer than $180 \mathrm{~ms}$, as a function of time is shown in Figure 7(a). The filament voltage of the electron emitter is shown in Figure $7(\mathrm{~b})$ and the CubeSat floating potential, which was measured by a multimeter, is shown in Figure 7(c). Before $250 \mathrm{~s}$, the electron emitter was off. When the m-NLP probes were not biased, the CubeSat potential was around $-0.6 \mathrm{~V}$. At the time the swept-bias mode started, the CubeSat floating potential became more negative and reached around $-4 \mathrm{~V}$ as long as the biases of the probes were at $10 \mathrm{~V}$. After $250 \mathrm{~s}$, the electron emitter was turned on with the filament voltage of $1.2 \mathrm{~V}$. The CubeSat potential became less negative and relatively stabilize around $-0.3 \mathrm{~V}$ when the m-NLP probes were not in operation. The CubeSat floated at about $-3 \mathrm{~V}$ as the probes were biased at $10 \mathrm{~V}$. It is noted that the situation where the probes are all biased at $10 \mathrm{~V}$ is an extreme case. The CubeSat potential could be less negative for the m-NLP probes biased at smaller positive voltages. The CubeSat potential variations are superimposed over one complete period of the probe bias sweep for the two cases: with and without using the electron emitter as shown in Figure 8. As can be seen, the CubeSat potential started to be affected by the sweeping probes when the biases were higher than 1 $\mathrm{V}$.

Figure 9 shows the electron density and CubeSat floating potential estimated by the $\mathrm{m}-\mathrm{NLP}$ system running in the fixed-bias mode. The probes were biased at different fixed 


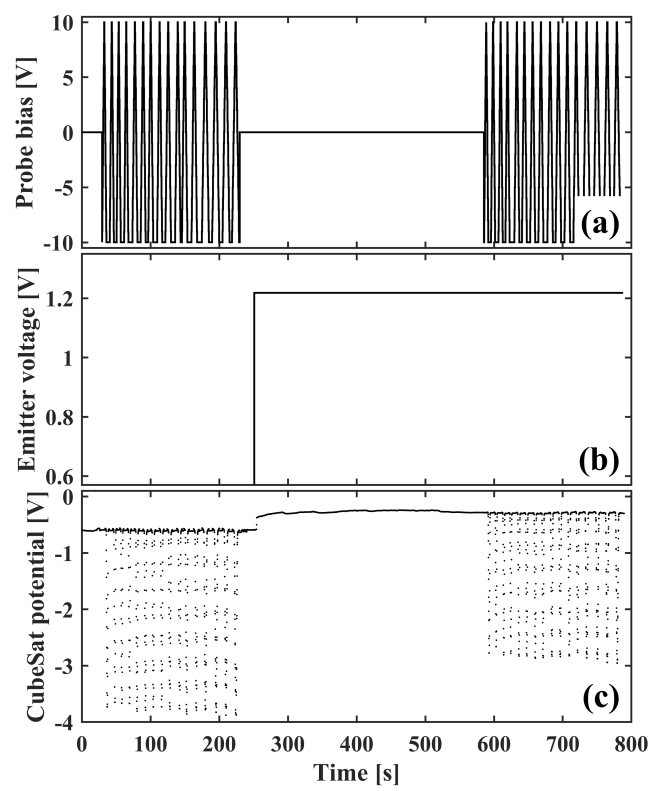

Fig. 7 Spacecraft charging mitigation by the electron emitter. (a) All the probes of m-NLP are swept simultaneously from $-10 \mathrm{~V}$ to $10 \mathrm{~V}$ and back to $-10 \mathrm{~V}$. (b) Filament voltage of the electron emitter. (c) CubeSat floating potential.

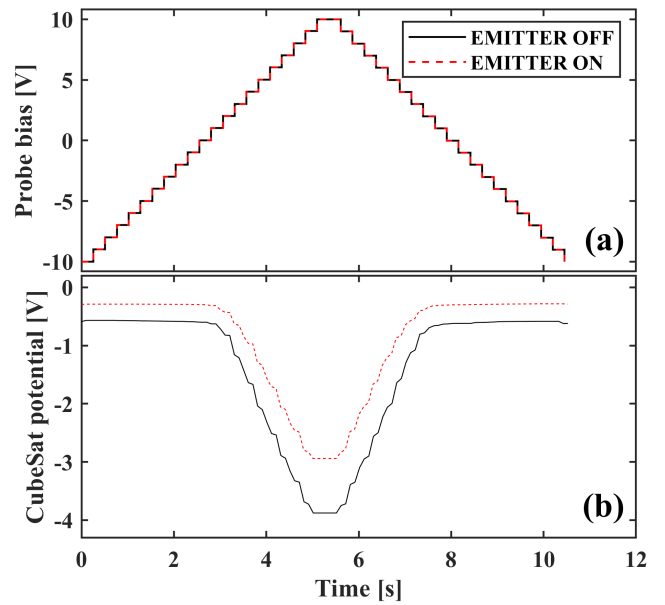

Fig. 8 The CubeSat potential variations shown in Figure 7 are superimposed over one complete period of the probe bias sweep for the two cases: with and without using the electron emitter. (a) Probe bias. (b). CubeSat potential. 


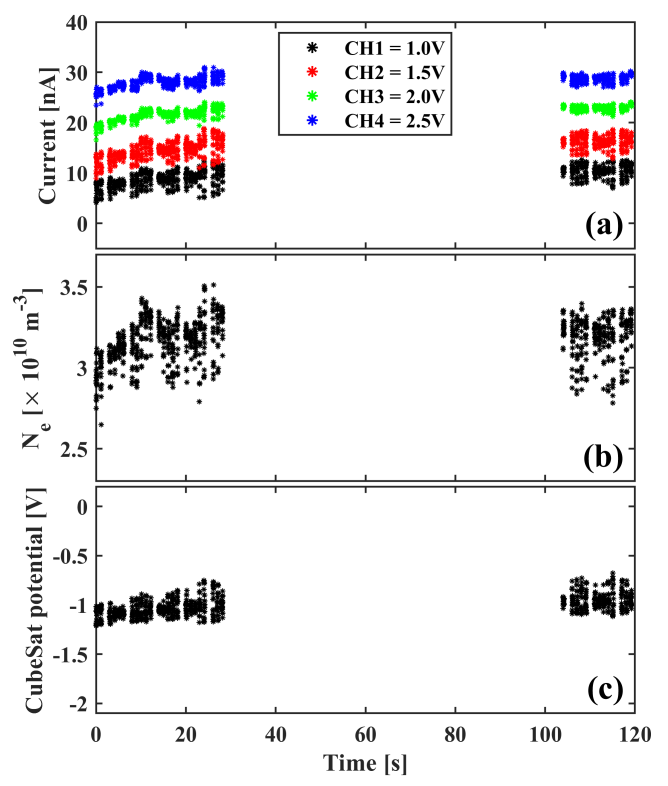

Fig. 9 Measurements in the fixed-bias mode of the m-NLP instrument. (a) Currents collected by the m-NLP probes. (b) Estimated electron density. (c) Estimated CubeSat potential. The electron density was calculated using Eq. 2 (having a linear fit to the square of the probe currents) and the floating potential was calculated using a method described in Bekkeng et al (2013) and Bekkeng et al (2017).

voltages $1.0 \mathrm{~V}, 1.5 \mathrm{~V}, 2.0 \mathrm{~V}$ and $2.5 \mathrm{~V}$. During the measurement period, the electron emitter was turned on with the filament voltage of $1.2 \mathrm{~V}$. The currents collected by the m-NLP probes are shown in Figure 9(a). Figure 9(b)-(c) show the calculated electron density and CubeSat floating potential, respectively. The electron density and CubeSat floating potential were roughly estimated to be $3-3.5 \times 10^{10} \mathrm{~m}^{-3}$ and to be $-1 \mathrm{~V}$, respectively, during the measurement process. The electron density was calculated using Eq. 2 (having a linear fit to the square of the probe currents) and the floating potential was calculated using a method described in Bekkeng et al (2013) and Bekkeng et al (2017). It is noted that the above values are just coarse estimates since the plasma environment in the chamber at the time of the measurements was believed to be inhomogeneous, meaning that the plasma at the probe locations might differ from each other. As can be seen from Figure 9, the measurements were only taken before $30 \mathrm{~s}$ and after $100 \mathrm{~s}$. Though there are some uncertainties in the plasma parameter estimation, the testing results have validated the m-NLP system operation and the electron emitter, which is capable of mitigating the spacecraft charging issue.

\section{First results and discussions}

Preliminary in-orbit data from two satellites, Ex-Alta 1 and Hoopoe, of the QB50 constellation are reported in this paper. Ex-Alta 1 and Hoopoe are 3U and $2 \mathrm{U}$ Cubesats developed by University of Alberta, Canada and Space Laboratory of the Herzliya Science Center, Israel, respectively. They were both launched on May 18, 2017 from the International Space Station (ISS). Due to the low bandwidth and limited power on the two satellites, the m-NLP instrument has been in operation only a few times since its launch into orbit. 


\subsection{Ex-Alta 1 case study}

Results of the STM experiment on Ex-Alta 1 recorded on October 21st, 2017 from 11:00 13:15 UT are shown in Figure 10(a), where temperatures measured by the six transducers, TH0 to Th5, are presented. Figure 10(b) shows the satellite location and altitude. The black dashed lines indicate the boundaries of nighttime region and the black dotted lines indicate the boundaries of civil twilight. As can be seen from Figure 10(a) all of the temperatures reduced by about $30{ }^{\circ} \mathrm{C}$ when the satellite flew from the dayside into the nightside. While the temperature inside the m-NLP electronics board TH5 is the highest of about $40^{\circ} \mathrm{C}$ on the dayside, the transducer TH0 located on the bottom panel ( $+\mathrm{Z}$ face) reports the lowest temperature on the dayside of just about $15{ }^{\circ} \mathrm{C}$. The nightside temperatures of these two transducers are about $9{ }^{\circ} \mathrm{C}$ and $-19{ }^{\circ} \mathrm{C}$, respectively. The satellite attitude data are currently under calibration. When the calibration process is done, this would help us to know exactly relative position of the spacecraft side panels with respect to the Sun.

During the first commissioning phase of the satellite, the m-NLP probes were biased at $0.8 \mathrm{~V}, 1.4 \mathrm{~V}, 1.8 \mathrm{~V}$ and $2.2 \mathrm{~V}$ without using the electron emitter. This was done on August 15th, 2017 from 11:01 - 11:15 UT when the satellite was in eclipse. The results indicate almost no electron current collected by the probes probably due to highly negative spacecraft potential. Ten days later, the electron emitter was turned on and the m-NLP recorded data from 11:11 to 11:16 UT as shown in Figure 11. Even though the m-NLP instrument has the highest sampling rate of $255 \mathrm{~Hz}$ on the QB50 satellites, we have been using just $10 \mathrm{~Hz}$ on Ex-Alta 1 due to the low bandwidth. Currents from the four m-NLP probes, which were biased at $1.0 \mathrm{~V}, 1.5 \mathrm{~V}, 2.0 \mathrm{~V}$ and $2.5 \mathrm{~V}$, are shown in the Figure 11(a), where Figure 11(b) shows the satellite location and altitude. The filament voltage of the electron emitter was set at $1.2 \mathrm{~V}$ for $10 \mathrm{~min}$ before this measurement process. During the measurement period, the filament voltage reduced from $1.2 \mathrm{~V}$ to $1 \mathrm{~V}$ by several voltage steps. The pink dashed line in Figure 11(a) indicates the filament voltage, which are actual measured data stored as housekeeping data on the m-NLP system and are periodically transfered to the OBC. The filament voltage were intended to be reduced to $0.95 \mathrm{~V}$ at around 11:15:30 UT, however, the housekeeping data after about 11:15:00 UT have not been available.

In looking at Figure 11, the astute reader is confronted with several anomalies, e.g. the current from the $1.0 \mathrm{~V}$ probe is higher than that from the probes with higher bias. Since the attitude data are not available yet, it is difficult to understand what happened to the $\mathrm{m}$ NLP probes. There are three main possibilities: (i) some of probes were possibly damaged during the satellite launch such that the surfaces of the probes are not uniform (ii) the probes were affected by plasma wakes (iii) the probes were not connected to the electronics in the right order. The other possibilities could be due to that photoemission from the four probes may be different and/or electrons emitted from the emitter may be recollected by the probes and contaminate the measurement. It is not possible to estimate the electron density and spacecraft potential with the data anomaly. However, a decrease in the probe current is observed as the MTEE filament voltage is lowered. This is a first in-flight demonstration of the MTEE, showing its capability to help mitigate the negative charging of the platform.

\subsection{Hoopoe case study}

Due to limited onboard memory of the Hoopoe satellite, the m-NLP binary script is limited to up to $2 \mathrm{~KB}$. This not only limits the sampling rate of the m-NLP science unit, but also the measurement time. Results of the STM experiment on Hoopoe recorded on October 


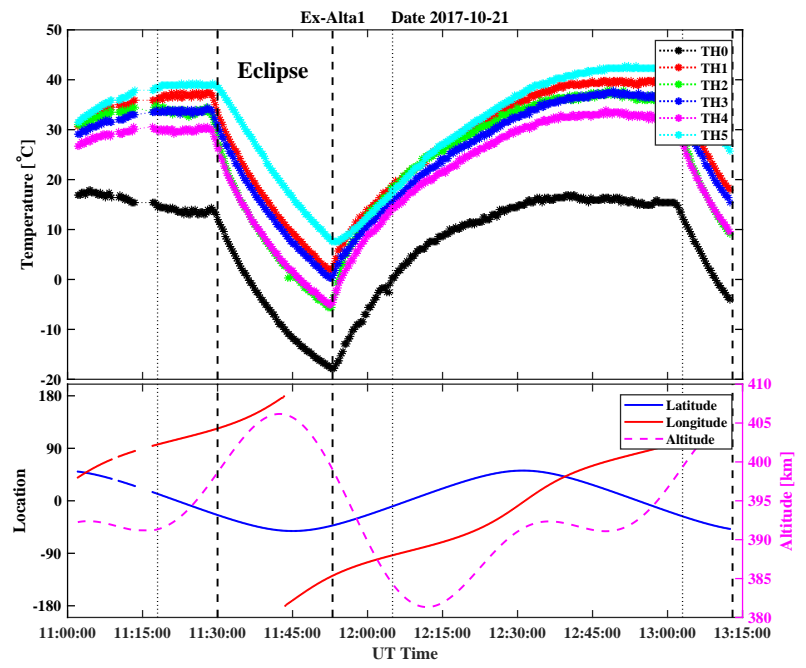

Fig. 10 Measured temperatures by the STM experiment on Ex-Alta 1. While the black dashed lines indicate the boundary of nighttime region, the black dotted lines indicate the boundary of civil twilight. (a) Measured temperatures. (b) Satellite location (left axis) and altitude above WGS84 (right axis).

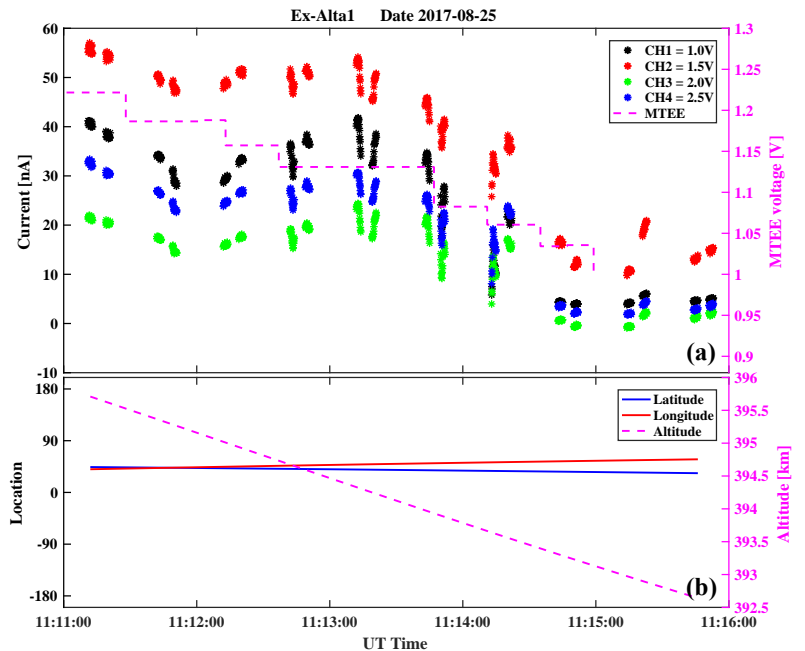

Fig. 11 Measurement data from the m-NLP instrument on Ex-Alta 1 on August 25th, 2017. (a) Currents collected by the m-NLP probes (left axis) and MTEE filament voltage (right axis). (b) Satellite location (left axis) and altitude above WGS84 (right axis).

21st, 2017 for 4 minutes from 11:04 - 11:08 UT are shown in Figure 12, where Figure 12(a) shows the measured temperatures and Figure 12(b) shows the satellite location and altitude. The experiment was done when the satellite was in eclipse. The m-NLP electronics board temperature is also the highest at about $8{ }^{\circ} \mathrm{C}$. Because of the lack of satellite attitude information, we cannot further interpret the data.

Similar to the Ex-Alta 1 case, the m-NLP probes biased at $0.8 \mathrm{~V}, 1.4 \mathrm{~V}, 1.8 \mathrm{~V}$ and $2.2 \mathrm{~V}$ collected almost no electron current without the electron emitter. The emitter was later used 


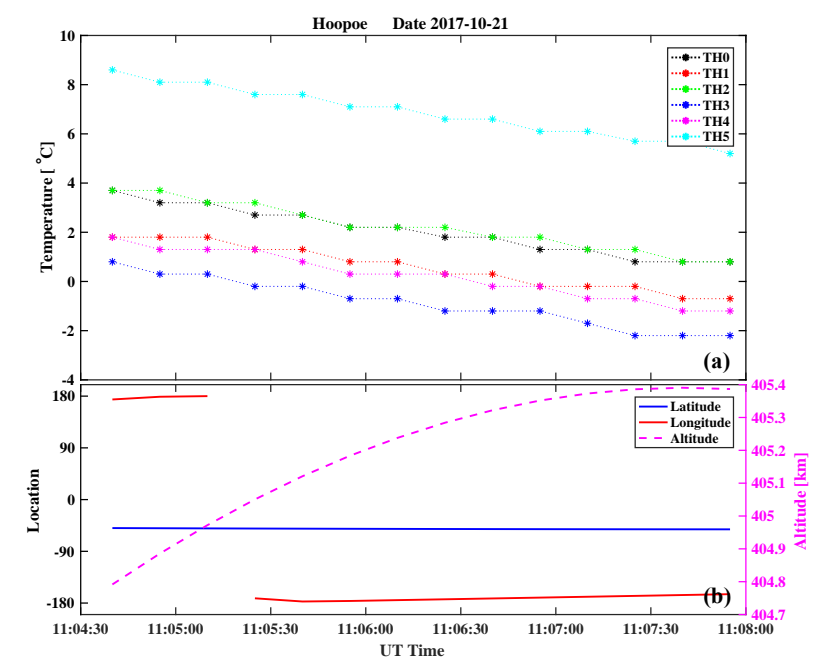

Fig. 12 Measured temperatures by the STM experiment on Hoopoe. The measurements were done when the satellite was in eclipse. (a) Measured temperatures. (b) Satellite location (left axis) and altitude above WGS84 (right axis).

during probe operation. The m-NLP measurement data recorded on October 21st, 2017 are shown in Figure 13. Because of a likely problem during the boom deployment, only probe 4 has been used in the operation. Thus it is not possible to estimate the spacecraft floating potential. However, the electron density can be roughly estimated as $N_{e} \simeq \frac{1}{K A_{e}} \frac{I_{e}}{\sqrt{V_{b}}}$, which is an approximation of the Eq. 1 for a single probe with high bias. Probe 4 was then biased at $10 \mathrm{~V}$ and the sampling rate was set at $10 \mathrm{~Hz}$. The MTEE filament voltage was at $1.2 \mathrm{~V}$ for 10 min before this measurement process. During the measurement period, the filament voltage was intended to reduce from $1.2 \mathrm{~V}$ to $0.95 \mathrm{~V}$, however, the housekeeping data indicate only a reduction down to $1 \mathrm{~V}$. The housekeeping data after 11:15 UT are missing, thus we are not sure if the MTEE followed the script setting. The electron density can be roughly estimated about $1-2.5 \times 10^{11} \mathrm{~m}^{-3}$ as shown in Figure 13(b), which also shows the electron density derived by the International Reference Ionosphere (IRI-2016) model. The IRI model is an empirical model providing estimates of ionospheric parameters from an altitude of 50 to $2000 \mathrm{~km}$ (Bilitza et al, 2014, 2017). It can be seen that, the electron density calculations of the two approaches are similar. The satellite location and altitude are shown in Figure 13(c). There was also a decrease in the measurement current as the MTEE voltage decreased as shown in Figure 13(a). The lower the MTEE filament voltages, the more negative the spacecraft floating potential causing the reduction in the electron current. The reader could think that the reduced current could be due to a reduction in the electron density since the satellite traveled over $1000 \mathrm{~km}$ during the measurement period. However, at the time the satellite was traveling towards the equator where the electron density was expected to be higher as estimated by IRI as shown in Figure 13(b). This has further validated the MTEE operation in orbit. It is noted that unlike the m-NLP technique, the electron density estimated by the single probe is dependent on the spacecraft potential, i.e. the more negative spacecraft potential the lower the estimated electron density. 


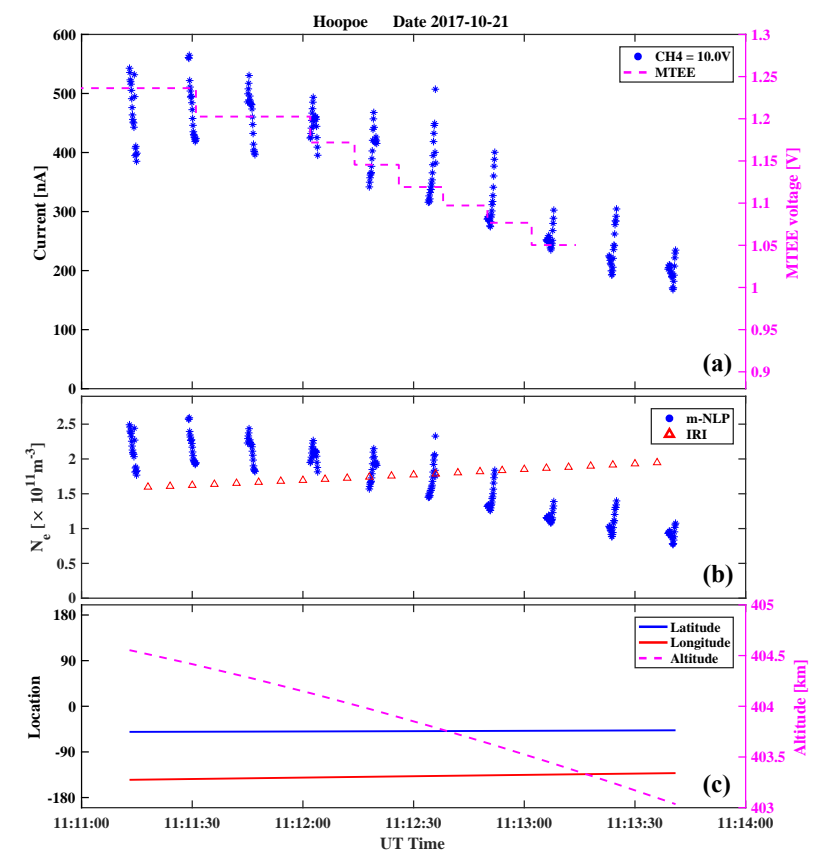

Fig. 13 Measurement data from the m-NLP instrument on Hoopoe on October 21st, 2017. (a) Current collected by the $10 \mathrm{~V}$ probe (left axis) and MTEE filament voltage (right axis). (b) Comparison between the electron density estimated by the $10 \mathrm{~V}$ probe and that estimated by the IRI model. (c) Satellite location (left axis) and altitude above WGS84 (right axis).

\section{Conclusion}

The multi-needle Langmuir probe (m-NLP) developed for the QB50 mission has been described in detail. The system functional verification has been successfully performed in the plasma chamber. The in-orbit preliminary data from two of the satellites in the QB50 constellation, Ex-Alta 1 and Hoopoe, have been shown, indicating that even though there were some uncertainties with the m-NLP measurements, some optimistic in-orbit preliminary results, which could be helpful for the system improvement in future campaigns, have been obtained. A first in-flight demonstration of the MTEE has been presented, showing a dependency on the MTEE filament voltage for the probe currents in both case studies. Furthermore, the temperatures on all of the satellite panels were observed, showing nominal values as expected. Work is in progress to resolve remaining uncertainties. Additionally, the spacecraft attitude information on the satellites has not been available, making it hard to interpret collected data, not only for probe behavior analysis but also for physical process study.

There are some remarks to be made about this campaign. First, with their really small diameter, the m-NLP probes are delicate and vulnerable to any vibration or shock during the delivery and launch process, which could possibly affect the probe surface uniformity. Second, while the great advantage of the m-NLP instrument is the ability to resolve plasma density with high resolution, due to the relatively low down-link capacity of the QB50 mission, the m-NLP sampling rate has been set at a very low level. Last but not least, in order to interpret the m-NLP data, accurate spacecraft attitude information is needed and this has not been achieved yet in the mission. Moving forward, spacecraft bus and boom design 
changes should be considered for the optimal m-NLP science unit deployment. Although the electron emitter is included in the unit, increasing the minimum conductive surface area of the spacecraft to be on the order of $200-300 \mathrm{~cm}^{2}$ is recommended in order to more effectively operate the electron emitter, which balances the electron current collected by the $\mathrm{m}$-NLP probes. This would also help to reduce the power consumption from the electron emitter. In addition, the boom system design can be adjusted so that the m-NLP probes are similarly oriented with respect to the magnetic field in order to mitigate likely impacts of a bi-Maxwellian plasma (Jacobsen et al, 2010). It is important to have a detection mechanism in the next version of the m-NLP system to make sure whether the boom deployment is succesfull. Particle-In-Cell (PIC) simulation is also needed to do modeling the coupling between between the m-NLP probes, the spacecraft and the plasma. This would give a better understanding of how the probe current collections are affected by plasma wakes and spacecraft sheath expansion.

The QB50 mission is an excellent opportunity for the involved universities to develop their own space programs and to demonstrate both space instruments and measurement techniques in orbit. The mission has enabled us to develop the electron emitter, which is then used to actively control the spacecraft platform potential. This makes the m-NLP available on many spacecraft for ionospheric density measurements.

Acknowledgements This research has received financial support from Research Council of Norway project 208006, 230996 and ESA PRODEX project 4200090335. The research leading to these results has also received funding from the European Research Council under the European Union's Seventh Framework Programme (FP7/2007-2013)/ ERC grant agreement 284427. The work is also a part of the 4DSpace initiative at the University of Oslo. Ex-Alta-1 was carried out with the financial support of donors, two crowd-sourcing campaigns, from the Canadian Space Agency, and from the Canadian Natural Sciences and Engineering Research Council (NSERC) through a Discovery Grant to Ian R. Mann.

\section{References}

Basu S, Basu S (1981) Equatorial scintillations-a review. Journal of Atmospheric and Terrestrial Physics 43(5):473 - 489, DOI https://doi.org/10.1016/0021-9169(81)90110-0, URL http://www.sciencedirect.com/science/article/pii/0021916981901100, equatorial Aeronomy - I

Basu S, Basu S, MacKenzie E, Fougere PF, Coley WR, Maynard NC, Winningham JD, Sugiura M, Hanson WB, Hoegy WR (1988a) Simultaneous density and electric field fluctuation spectra associated with velocity shears in the auroral oval. Journal of Geophysical Research: Space Physics 93(A1):115-136, DOI 10.1029/JA093iA01p00115, URL http://dx.doi.org/10.1029/JA093iA01p00115

Basu S, MacKenzie E, Basu S (1988b) Ionospheric constraints on vhf/uhf communications links during solar maximum and minimum periods. Radio Science 23(03):363-378, DOI 10.1029/RS023i003p00363

Basu S, Basu S, MacKenzie E, Coley WR, Sharber JR, Hoegy WR (1990) Plasma structuring by the gradient drift instability at high latitudes and comparison with velocity shear driven processes. Journal of Geophysical Research: Space Physics 95(A6):7799-7818, DOI 10.1029/JA095iA06p07799, URL http://dx.doi.org/10.1029/JA095iA06p07799

Basu S, Weber EJ, Bullett TW, Keskinen MJ, MacKenzie E, Doherty P, Sheehan R, Kuenzler H, Ning P, Bongiolatti J (1998) Characteristics of plasma structuring in the cusp/cleft region at svalbard. Radio Science 33(6):1885-1899, DOI 10.1029/98RS01597, URL http://dx.doi.org/10.1029/98RS01597 
Beattie AM, Helleren $\varnothing$, Zee RE (2013) In-flight operations of a high-availability nanosatellite constellation for maritime observation. In: Proceedings of the 64th International Astronautical Congress (IAC 2013), URL http://utias-sfl.net/wpcontent/uploads/International-Astronautical-Congress-2013-IAC-13B431x16514.pdf

Bekkeng TA (2017) Development of a miniaturized multi-needle langmuir probe system for in-situ measurements of electron density and spacecraft floating potential. $\mathrm{PhD}$ thesis, University of Oslo

Bekkeng TA, Jacobsen KS, Bekkeng JK, Pedersen A, Lindem T, Lebreton JP, Moen JI (2010) Design of a multi-needle langmuir probe system. Measurement Science and Technology 21(8):085,903, URL http://stacks.iop.org/0957-0233/21/i=8/a=085903

Bekkeng TA, Barjatya A, Hoppe UP, Pedersen A, Moen JI, Friedrich M, Rapp M (2013) Payload charging events in the mesosphere and their impact on langmuir type electric probes. Annales Geophysicae 31(2):187-196, DOI 10.5194/angeo-31-187-2013, URL http://www.ann-geophys.net/31/187/2013/

Bekkeng TA, Helgeby ES, Pedersen A, Trondsen E, Lindem T, Moen JI (2017) multi-needle langmuir probe system for electron density measurements and active spacecraft potential control on cubesats, submitted to IEEE Transactions on Aerospace and Electronic Systems

Bilitza D, Altadill D, Zhang Y, Mertens C, Truhlik V, Richards P, McKinnell LA, Reinisch B (2014) The international reference ionosphere 2012 - a model of international collaboration. Journal of Space Weather and Space Climate 4:A07, DOI 10.1051/swsc/2014004, URL https://doi.org/10.1051/swsc/2014004

Bilitza D, Altadill D, Truhlik V, Shubin V, Galkin I, Reinisch B, Huang X (2017) International reference ionosphere 2016: From ionospheric climate to real-time weather predictions. Space Weather 15(2):418-429, DOI 10.1002/2016SW001593, URL http://dx.doi.org/10.1002/2016SW001593, 2016SW001593

Boggess RL, Brace LH, Spencer NW (1959) Langmuir probe measurements in the ionosphere. Journal of Geophysical Research 64(10):1627-1630, DOI 10.1029/JZ064i010p01627, URL http://dx.doi.org/10.1029/JZ064i010p01627

Brace LH (1998) Langmuir Probe Measurements in the Ionosphere, in Measurement Techniques in Space Plasmas:Particles. American Geophysical Union, DOI 10.1029/GM102p0023

Brace LH, Theis RF, Dalgarno A (1973) The cylindrical electrostatic probes for atmosphere explorer -c,-d, and -e. Radio Science 8(4):341-348, DOI 10.1029/RS008i004p00341, URL http://dx.doi.org/10.1029/RS008i004p00341

Chapkunov SK, Ivanova TN, Petrunova MK, Serafimov KB (1976) Measurement of electron and ion density and temperature on the intercosmos 12 satellite. In: Space research XVI; Proceedings of the Open Meetings of Working Groups on Physical Sciences, pp 423-425

Chen FF (1965) Electric probes, Academic Press, chap 4, pp 113-200

Denis A (2015) QB50 System Requirements and Recommendations. URL https://qb50.eu/index.php/tech-docs/category/25-up-to-date-docs?download=89:qb50docs

Goodwin LV, Iserhienrhien B, Miles DM, Patra S, van der Meeren C, Buchert SC, Burchill JK, Clausen LBN, Knudsen DJ, McWilliams KA, Moen J (2015) Swarm in situ observations of $\mathrm{f}$ region polar cap patches created by cusp precipitation. Geophysical Research Letters 42(4):996-1003, DOI 10.1002/2014GL062610, URL http://dx.doi.org/10.1002/2014GL062610, 2014GL062610

Helleren Ø, Olsen Ø, Narheim BT, Skauen AN, Olsen RB (2012) Aissat-1 - 2 years of service. In: European Space Agency Small Satellite Systems and Services Symposium 
Hoang H, Røed K, Bekkeng TA, Trondsen E, Clausen LBN, Miloch WJ, Moen JI (2017) High spatial-resolution electron density measurement by langmuir probe for multipoint observations using tiny spacecraft. Measurement Science and Technology URL http://iopscience.iop.org/10.1088/1361-6501/aa87e1

Hoang H, Røed K, Bekkeng TA, Moen JI, Spicher A, Clausen LBN, Miloch WJ, Trondsen E, Pedersen A (2018) A study of data analysis techniques for the multineedle langmuir probe. Measurement Science and Technology 29(6):065,906, URL http://stacks.iop.org/0957-0233/29/i=6/a=065906

Jacobsen KS, Pedersen A, Moen JI, Bekkeng TA (2010) A new langmuir probe concept for rapid sampling of space plasma electron density. Measurement Science and Technology 21(8):085,902, URL http://stacks.iop.org/0957-0233/21/i=8/a=085902

Kintner PM, Ledvina BM, de Paula ER (2007) Gps and ionospheric scintillations. Space Weather 5(9):n/a-n/a, DOI 10.1029/2006SW000260, URL http://dx.doi.org/10.1029/2006SW000260, s09003

Mann IR, et al (2018) The ex-alta 1 cubesat mission. Submitted to Space Science Reviews (this issue)

Miles DM, Bennest JR, Mann IR, Millling DK (2013) A radiation hardened digital fluxgate magnetometer for space applications. Geoscientific Instrumentation, Methods and Data Systems 2(2):213-224, DOI 10.5194/gi-2-213-2013, URL https:/www.geosci-instrummethod-data-syst.net/2/213/2013/

Miles DM, Mann IR, Ciurzynski M, Barona D, Narod BB, Bennest JR, Pakhotin IP, Kale A, Bruner B, Nokes CDA, Cupido C, Haluza-DeLay T, Elliott DG, Milling DK (2016) A miniature, low-power scientific fluxgate magnetometer: A stepping-stone to cube-satellite constellation missions. Journal of Geophysical Research: Space Physics 121(12):11,839_ 11,860, DOI 10.1002/2016JA023147, URL http://dx.doi.org/10.1002/2016JA023147, 2016JA023147

Moen J, Oksavik K, Abe T, Lester M, Saito Y, Bekkeng TA, Jacobsen KS (2012) First in-situ measurements of hf radar echoing targets. Geophysical Research Letters 39(7):n/a-n/a, DOI 10.1029/2012GL051407, URL http://dx.doi.org/10.1029/2012GL051407, 107104

Moen J, Oksavik K, Alfonsi L, Daabakk Y, Romano V, Spogli L (2013) Space weather challenges of the polar cap ionosphere. Journal of Space Weather and Space Climate DOI https://doi.org/10.1051/swsc/2013025

Mott-Smith HM, Langmuir I (1926) The theory of collectors in gaseous discharges. Physical Review 28:727-763, DOI 10.1103/PhysRev.28.727

Muylaert J, Reinhard R, Asma C, Danilkin V (2009) Qb50, an international network of 50 cubesats for multi-point, in-situ measurements in the lower thermosphere and re-entry research. In: QB50 Workshop

Oksavik K, Moen J, Lester M, Bekkeng TA, Bekkeng JK (2012) In situ measurements of plasma irregularity growth in the cusp ionosphere. Journal of Geophysical Research: Space Physics 117(A11):n/a-n/a, DOI 10.1029/2012JA017835, URL http://dx.doi.org/10.1029/2012JA017835, a11301

Prikryl P, Jayachandran PT, Mushini SC, Pokhotelov D, MacDougall JW, Donovan E, Spanswick E, St-Maurice JP (2010) Gps tec, scintillation and cycle slips observed at high latitudes during solar minimum. Annales Geophysicae 28(6):1307-1316, DOI 10.5194/angeo-28-1307-2010, URL https://www.ann-geophys.net/28/1307/2010/

Program CPSTC (2015) Cubesat design specification

Spicher A, Miloch WJ, Moen JI (2014) Direct evidence of double-slope power spectra in the high-latitude ionospheric plasma. Geophysical Research Letters 41(5):1406-1412, DOI 10.1002/2014GL059214, URL http://dx.doi.org/10.1002/2014GL059214 
Spicher A, Cameron T, Grono EM, Yakymenko KN, Buchert SC, Clausen LBN, Knudsen DJ, McWilliams KA, Moen JI (2015a) Observation of polar cap patches and calculation of gradient drift instability growth times: A swarm case study. Geophysical Research Letters 42(2):201-206, DOI 10.1002/2014GL062590, URL http://dx.doi.org/10.1002/2014GL062590, 2014GL062590

Spicher A, Miloch WJ, Clausen LBN, Moen JI (2015b) Plasma turbulence and coherent structures in the polar cap observed by the ici-2 sounding rocket. Journal of Geophysical Research: Space Physics 120(12):10,959-10,978, DOI 10.1002/2015JA021634, URL http://dx.doi.org/10.1002/2015JA021634, 2015JA021634

Venturini C, Abramowitz L, Johansen J, Gee J, Floyd W (2009) Cubesat developmental programs-working with the community. In: AIAA SPACE 2009 Conference \& Exposition, AIAA-6501, DOI 10.2514/6.2009-6501 